\title{
THE NATURE AND IMPACT OF ZNO BUFFER LAYERS ON THE INITIAL STAGES OF THE HYDRIDE VAPOR PHASE EPITAXY OF GAN
}

\author{
Shulin Gu, Rong Zhang, Jingxi Sun, Ling Zhang, and T. F. Kuech \\ Department of Chemical Engineering, University of Wisconsin, \\ Madison, WI 53706
}

\begin{abstract}
The nature and impact of $\mathrm{ZnO}$ buffer layers on the initial stages of the hydride vapor phase epitaxy (HVPE) of GaN have been studied by xray photoelectron spectroscopy (XPS), atomic force microscopy (AFM), xray diffraction (XRD) and photoluminescence (PL). During pre-growth heating, the surface $\mathrm{ZnO}$ layer was found to both desorb from $\mathrm{ZnO}$-coated sapphire and react with the underlying sapphire surface forming a thin $\mathrm{ZnAl}_{2} \mathrm{O}_{4}$ alloy layer between $\mathrm{ZnO}$ and sapphire surface. This $\mathrm{ZnO}$-derived surface promotes the initial nucleation of the $\mathrm{GaN}$ and markedly improves material surface morphology, quality and growth reproducibility.
\end{abstract}

\section{Introduction}

Hydride Vapor Phase Epitaxy (HVPE) is a promising technique to grow thick $\mathrm{GaN}$ materials. These thick layers can be used in device applications as well as a substrate for subsequent low-defect GaN growth by Metal-organic Vapor Phase Epitaxy (MOVPE) [1,2]. Due to the large lattice mismatch, the initial nucleation and growth of $\mathrm{GaN}$ on sapphire substrates determines the material properties of the subsequent epitaxial layer. In MOVPE, low temperature GaN or AlN buffer layers have been used to improve this initial nucleation for the subsequent high temperature GaN growth $[3,4]$. In the case of the HVPE process, low temperature buffer layers exhibit poor crystalline quality and lead to the deposition of polycrystalline material at high growth temperatures [5]. The initial nucleation behavior on sapphire, however, can be improved by the inclusion of a $\mathrm{ZnO}$ intermediate or buffer layer $[1,2,5,6]$. Based plane $\mathrm{ZnO}$ could provide a better lattice match between $\mathrm{GaN}$ and sapphire. In many cases, the $\mathrm{ZnO}$ buffer layer has been reported not to survive the initial heating and pre-growth treatment and is not reported to be present in the final epitaxial multi-layer structure [5]. Attempts to deposit highest quality $\mathrm{ZnO}$ buffer layer to avoid its dissociation from sapphire surface do not lead to further improvements in material quality but rather exhibit poorer properties in the subsequent $\mathrm{GaN}$ layer. In this case, some $\mathrm{ZnO}$ has been noted at the interface between the high temperature $\mathrm{GaN}$ and the sapphire [6]

The mechanism underlying the impact of the $\mathrm{ZnO}$ buffer layer on the initial stages of HVPE of GaN material is unknown, particularly in the absence of $\mathrm{ZnO}$ in the final structure $[3,5,6]$. $\mathrm{ZnO}$ can reactively diffuse into $\mathrm{Al}_{2} \mathrm{O}_{3}$ forming a spinel of $\mathrm{ZnAl}_{2} \mathrm{O}_{4}$ at high tempaeratures [7-9]. In previous studies, reactions between $\mathrm{ZnO}$ and $\mathrm{Al}_{2} \mathrm{O}_{3}$ powders, were limited in the absence of oxygen [9]. In this case, the gas phase transport of $\mathrm{ZnO}$ and its stability is affected by the oxygen activity. There is little data on the interdiffusion between epitaxial $\mathrm{ZnO}$ and sapphire. $\mathrm{ZnAl}_{2} \mathrm{O}_{4}$ surface layer, if present at the 
growth front, could improve nucleation by lowering the surface energy and could provide an improved lattice match. In this paper, we have studied the formation, annealing and structure of $\mathrm{ZnO}$ epitaxial layers on sapphire used to promote nucleation and improve the subsequent $\mathrm{GaN}$ growth. In particular, the structure and composition of the $\mathrm{ZnO}$-sapphire interface after high temperature annealing was investigated by atomic force microscopy (AFM), reflection high energy electron diffraction (RHEED), x-ray photoelectron spectroscopy (XPS), and x-ray diffraction (XRD) measurements.

\section{Experiment Detail}

$\mathrm{ZnO}$ buffer layers were initially deposited on sapphire surface by RF diode sputtering. Prior to $\mathrm{ZnO}$ deposition, the bare sapphire wafer was degreased with acetone and methanol followed by etching in a 3:1 sulfuric:phosphoric acid solution at $100^{\circ} \mathrm{C}$ and a final deionized water rinse. The wafer is then loaded into a RF diode sputtering system employing deposition conditions similar to those reported by Molnar et al [2]. $\mathrm{ZnO}$ thickness varied from $20 \mathrm{~nm}$ to $200 \mathrm{~nm}$ depending on the deposition time. RHEED measurements show this as-deposited $\mathrm{ZnO}$ film has a high degree of structural orientation. The FWHM of the XRD (002) rocking curves of these films was about 1.3 degrees. Prior to loading in the HVPE reactor, the $\mathrm{ZO}$ coated wafer was again ultrasonically cleaned in acetone and methanol.

$\mathrm{GaN}$ was grown on these wafers in a vertical HVPE reactor. The interior structure of the reactor for gas distribution is similar to that of the horizontal system previously reported $[10,11]$. In the present system, an additional region called a backflow tube was added which allowed the sample to be heated to the growth temperature under a $\mathrm{N}_{2}$ flow. After loading into the reactor, the $\mathrm{ZnO}$-coated wafer was preheated to the growth temperature in the backflow tube for 10 mins under a flowing N2 environment and then introduced to the flowing growth stream containing the $\mathrm{GaCl}$ and $\mathrm{NH}_{3}$ to initiate growth. The flow rate of $\mathrm{HCl}$ to Ga source controlled the growth rate over a range of 0.5 to 4 $\mu \mathrm{m} / \mathrm{min}$ in this work.

\section{Results and discussion}

$\mathrm{ZnO}$ buffer
layer will reactively dissociate under the HVPE pre-growth annealing conditions, leaving a specular surface since it is

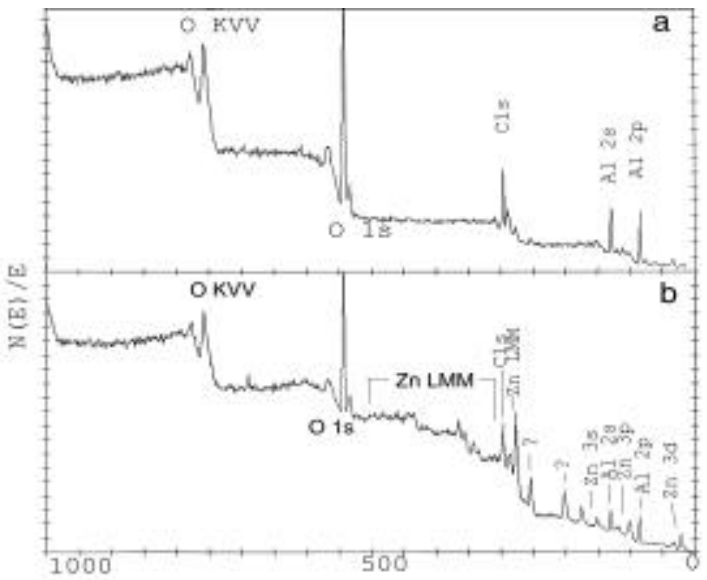

Fig.1 XPS spectra of Zn-derived sapphire surface after heat-treated under normal pre-growth conditions, (a) removing $\mathrm{ZnO}$ layer prior to annealing, and (b) removing $\mathrm{ZnO}$ layer after annealing. 
thermodynamically unstable within an oxygen-free ambient. For buffer layer greater than $20 \mathrm{~nm}$, PL and XRD studies reveal a residual film remains on sapphire surface despite an anneal time sufficient to dissociate this $\mathrm{ZnO}$ layer. The thickness of this residual layer depends on the initial $\mathrm{ZnO}$ thickness and annealing time. The chemical composition of the specular surface was initially examined by XPS. Prior to the XPS measurement, these samples receiving only the standard pre-growth conditions were dipped into an $\mathrm{HCl}$ solution to insure removal of any residual $\mathrm{ZnO}$ layer, if present. $\mathrm{ZnO}$ is readily etched in $\mathrm{HCl}$. XPS measurements confirm the formation of a thin residual $\mathrm{Zn}$-containing layer on the sapphire surface which is not $\mathrm{ZnO}$. As a contrast, removal of the $\mathrm{ZnO}$ layer from sapphire surface prior to the pre-growth annealing results in no detectable $\mathrm{Zn}$ signal from the sapphire surface by XPS. These XPS results are shown in Fig.1, for both heat-treated under normal pre-growth conditions, spectrum (a) was obtained from the sample which has the $\mathrm{ZnO}$ layer removed prior to annealing and spectrum (b) from a sample etched in $\mathrm{HCl}$ after annealing. This figure indicates that some surface $\mathrm{Zn}$ remains as a result of a surface reaction that has occurred during pre-growth anneal, not during $\mathrm{ZnO}$ deposition process. Spectrum $b$ is very similar to that obtained from $\mathrm{ZnAl}_{2} \mathrm{O}_{4}$ formed by annealing mixed $\mathrm{ZnO}$ and $\mathrm{Al}_{2} \mathrm{O}_{3}$ powder at high temperatures [12], suggesting a thin $\mathrm{ZnAl}_{2} \mathrm{O}_{4}$ layer formed during annealing. XRD measurements on this sample were not sufficiently sensitive to detect such a thin layer. In order to confirm the presence of a surface $\mathrm{ZnAl}_{2} \mathrm{O}_{3}$ layer by $\mathrm{XRD}$, we annealed a thick $\mathrm{ZnO}$ layer on sapphire in air for $30 \mathrm{hrs}$. The XRD spectrum obtained from this sample shown in Fig.2, contains the (006) sapphire peak, as well as the (111) and (333) diffraction peaks of $\mathrm{ZnAl}_{2} \mathrm{O}_{4}$. $\mathrm{ZnAl}_{2} \mathrm{O}_{4}$ is a cubic spinel, and the presence of the (111) and (333) peaks indicates a degree of epitaxial alignment in this reactive diffusion couple. This welloriented surface layer will have the (111) texture desired for the subsequent $\mathrm{GaN}$ growth.

The surface reaction and compound formation modifies the

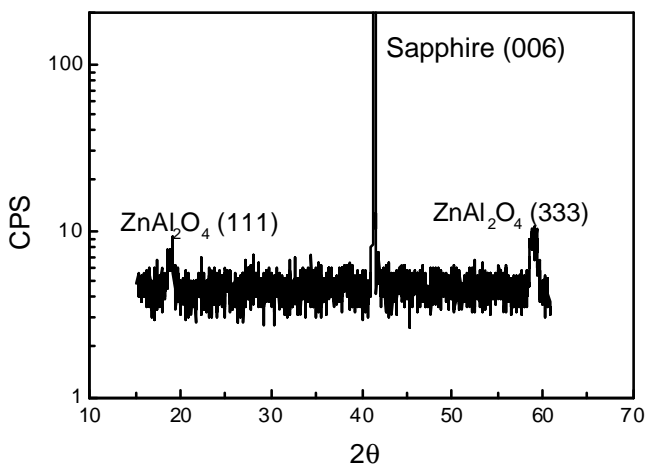

Fig. 2 XRD spectrum of Zn-derived sapphire surface annealing at air ambience and for $30 \mathrm{hrs}$.

sapphire surface morphology. Fig. 3 is the AFM micrograph of the samples used to obtain the spectrum of Fig.1. The surface of sample a has no obvious morphological modification due to the pre-growth anneal and is similar to the bare sapphire surface after annealing in a $\mathrm{N}_{2}$ ambient (not shown here). The surface of sample $\mathrm{b}$ is characterized by high density of small islands on the wafer surface. It is this ZnO-derived surface that promotes the enhanced initial nucleation and improved material quality in the subsequent $\mathrm{GaN}$ growth.

The surface reaction and compound formation modifies the sapphire surface morphology. Fig. 3 is the AFM micrograph of the samples used to obtain the spectrum of 

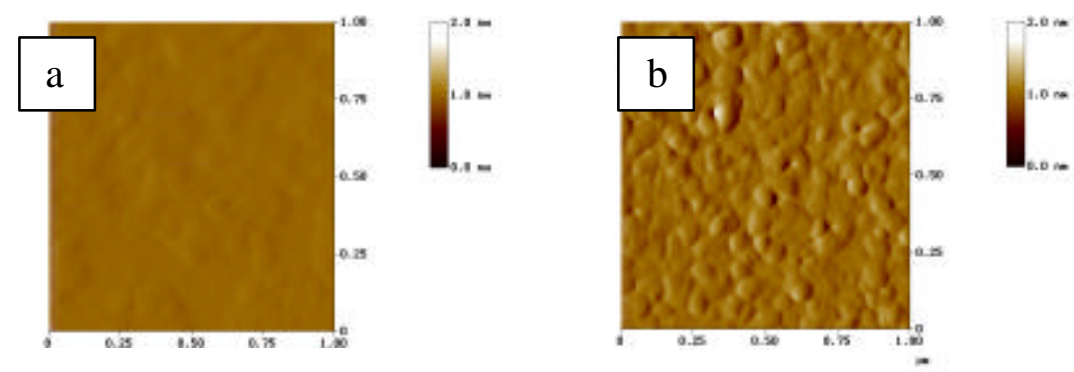

Fig.3 AFM micrograph of $\mathrm{Zn}$-derived sapphire surface, (a) removing $\mathrm{ZnO}$ prior to annealing, (b) removing $\mathrm{ZnO}$ after annealing

Fig.1. The surface of sample a has no obvious morphological modification due to the pre-growth anneal and is similar to the bare sapphire surface after annealing in a $\mathrm{N}_{2}$ ambient (not shown here). The surface of sample $b$ is characterized by high density of small islands on the wafer surface. It is this $\mathrm{ZnO}$-derived surface that promotes the enhanced initial nucleation and improved material quality in the subsequent GaN growth.
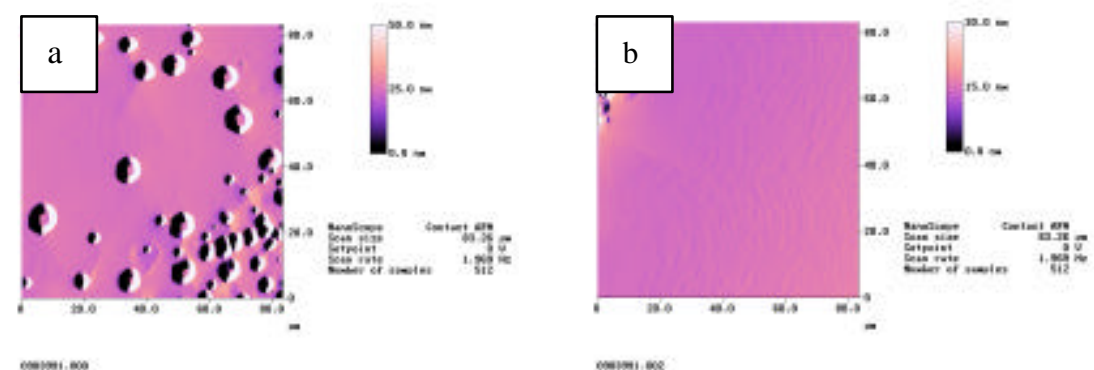

Fig.4 AFM micrograph of GaN material surface morphology (a) on bare sapphire, (b) on $20 \mathrm{~nm} \mathrm{ZnO}$ buffer layer

GaN growth on the bare sapphire, often leads to a rough surface with inferior material properties. It is often difficult to reproducibly obtain high quality $\mathrm{GaN}$ possessing a smooth surface, narrow XRD linewidth, and low carrier concentration. In contrast, the use of $\mathrm{ZnO}$ buffer layers, routinely leads to smoother surfaces with few morphology features as well as improved materials properties. Fig.4 contains AFM micrographs of GaN surfaces grown on (a) bare sapphire and (b) an initial $20 \mathrm{~nm} \mathrm{ZnO}$ buffer layer. The surface pit density is very high for GaN material grown on bare sapphire, which is often associated with the nucleation during initial stages of growth. A surface with the growth features related with step flow growth mode characterize the $\mathrm{GaN}$ layer grown on the $\mathrm{ZnO}$ buffer layer. XRD measurements on the GaN layers with an $8 \mu \mathrm{m}$ in thickness possess a (002) rocking curve lindwidth as 400 to 500 arcsec, compared with the GaN layers of a similar thickness grown on bare sapphire normally a linwidth of 700 arcsec or more. 
Room temperature PL spectra of GaN grown on bare sapphire, a $20 \mathrm{~nm}$ thick $\mathrm{ZnO}$ buffer layer, and a $80 \mathrm{~nm}$ thick buffer layer are shown in Fig.5. The PL spectrum of $\mathrm{GaN}$ layer on the $20 \mathrm{~nm}$ thick $\mathrm{ZnO}$ buffer layer has a slightly narrower bandage emission of 4 to $5 \mathrm{~nm}$ of FWHM compared to the FWHM of 7-8 nm for layers grown on bare sapphire. The $\mathrm{GaN}$ grown on the $\mathrm{ZnO}$ buffer layer also exhibits a weak blue band emission, which has been previously related to $\mathrm{Zn}$ doping $[1,6]$. This blue band emission centered at $420 \mathrm{~nm}$ increases in intensity in the PL spectrum of GaN material grown on a thick $80 \mathrm{~nm}$ buffer layer, also shown in Fig.5.

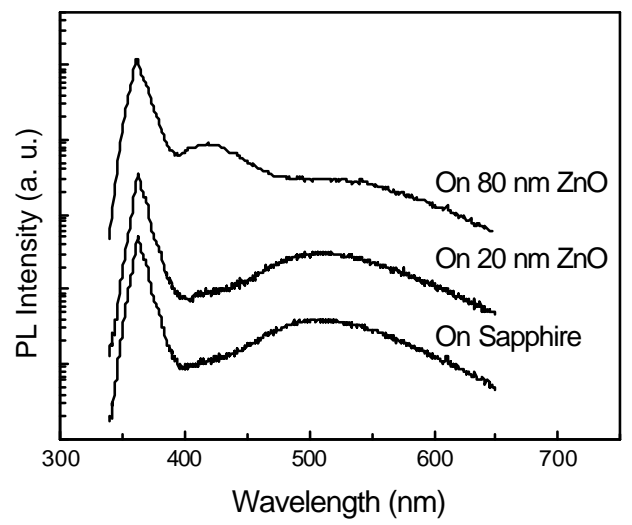

Fig.5 Room temperature PL spectra of $\mathrm{GaN}$ grown on different buffer layers

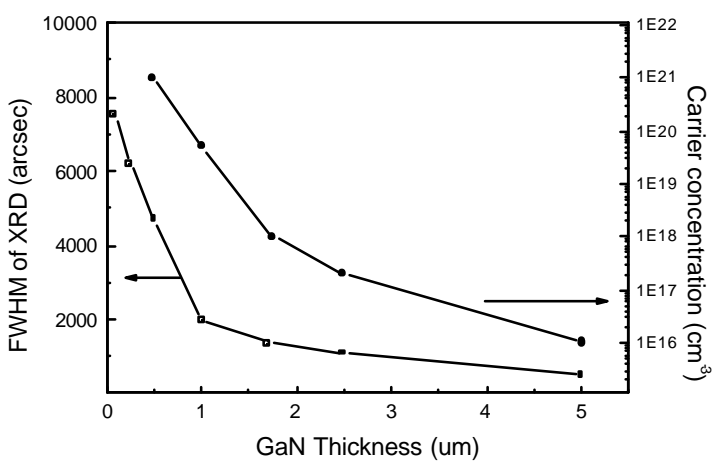

Fig. 6 FWHM of XRD (002) and carrier concentration of the $\mathrm{GaN}$ materials grown on $20 \mathrm{~nm} \mathrm{ZnO}$ buffer layer versus material thickness.
Growth on thick $\mathrm{ZnO}$ buffer layers can lead to delamination of the $\mathrm{GaN}$ layer from substrate, however, no such delamination occurs for materials grown on $20 \mathrm{~nm}$ buffer layer. Delamination is associated to the presence of a residual $\mathrm{ZnO}$ layer atop of the $\mathrm{ZnAl}_{2} \mathrm{O}_{4}$ layer which can persist after the pre-growth anneal for $\mathrm{ZnO}$ layers $80 \mathrm{~nm}$ or more in thickness. This residual $\mathrm{ZnO}$ layer is found to degrade the GaN quality, despite the potential to provide a better lattice matched layer. The line width of the $\mathrm{GaN}$ bandedge emission is broader than that found from $\mathrm{GaN}$ grown on the thin buffer layer $(10 \mathrm{~nm}$ or more, depending on growth conditions), as found in a previous study [6]. For our specific system and pregrowth anneal the optimum thickness of the $\mathrm{ZnO}$ buffer layer is about $20 \mathrm{~nm}$, which is thick enough to form the surface $\mathrm{ZnAl}_{2} \mathrm{O}_{4}$ layer but leaves no residual $\mathrm{ZnO}$ prior to starting the growth. Finally, the similar yellow band emissions, seen in Fig.5 for all three samples, is believed to be primarilly related to the specific growth conditions used in the high temperature GaN layers. The intensity of 
YL can be decreased by a factor of one hundred by changing the growth conditions.

The change in material properties with GaN thickness has also been studied on the optimized $\mathrm{ZnO}$ buffer layer as shown in Fig.6. The FWHM of XRD and carrier concentration determining from $\mathrm{CV}$ measurements decrease quickly with material thickness. For a $5 \mu \mathrm{m}$ thick $\mathrm{GaN}$ on $20 \mathrm{~nm}$ thick $\mathrm{ZnO}$ buffer layer, the FWHM of XRD is less than 500 arcsec with a surface carrier concentration as low as $1 \times 10^{16} / \mathrm{cm}^{3}$. The GaN surface after $5 \mu \mathrm{m}$ of growth is smooth with few morphology features. In contrast, $\mathrm{GaN}$ grown on bare sapphire exhibits a much slower drop in XRD linewidth and surface carrier concentration with thickness. To achieve a similar materials properties for $\mathrm{GaN}$ grown on bare sapphire a layer of $20 \mu \mathrm{m}$ or more is required in our growth system under similar growth conditions.

\section{Conclusion}

$\mathrm{ZnO}$ buffer layers provide significant improvements in $\mathrm{GaN}$ material quality and system reproducibility by the HVPE method. The formation of a $\mathrm{ZnAl}_{2} \mathrm{O}_{4}$ surface layer by reactive diffusion between the $\mathrm{ZnO}$ and sapphire during pre-growth annealing process, can improve the initial nucleation and lead to a more rapid improvement in the $\mathrm{GaN}$ properties with thickness over layers grown on bare sapphire under similar conditions. The presence of this thin epitaxial $\mathrm{ZnAl}_{2} \mathrm{O}_{4}$ layer has been confirmed by XPS and XRD measurements.

\section{Acknowledgement}

This work is supported by DARPA-EPRI program on high power GaN devices.

\section{References:}

[1] T. Detchprohm, K. Hiramatsu, H. Amano, and I. Akasaki, Appl. Phys. Lett., 61, 2688(1992).

[2] R. J. Molnar, K. B. Nichols, P. Maki, E. R. Brown, and I. Melngailis, Mater. Res. Soc. Symp. Proc., 378, 479 (1995).

[3] S. Nakamura, Jpn. J. Appl. Phys., 30, L1705 (1991).

[4] I. Akasaki, and H. Amano, J. Crystal Growth, 163, 86 (1996).

[5] R. J. Monlar, W. Gotz, L. T. Romano, and J. M. Johnson, J. Crystal Growth, 178, 147 (1997).

[6] T. Ueda, T. F. Huang, S. Spruytte, H. Lee, M. Yuri, K. Itoh, T. Baba, and J. S. Harris Jr, J. Crystal Growth, 187, 340 (1998).

[7] J. T. Keller, D. K. Agrawal, and H. A. Mckinstry, Advanced Ceramic Materials, 3(4), 420 (1988).

[8] S. K. Sampath, and J. F. Cordaro, J. Am. Ceram. Soc., 81(3), 649 (1998).

[9] D. L. Branson, J. Am. Ceram. Soc., 48(11), 591 (1965).

[10] N. R. Perkins, M. N. Horton, Z. Z. Bandic, T. C. McGill, and T. F. Kuech, Mat. Res. Soc. Symp. Proc., 395, 243 (1996).

[11] R. Zhang, and T. F. Kuech, Mat. Res. Soc. Symp. Proc., 482, 709 (1998).

[12] B. R. Strohmeier, Surface Science Spectra, 3(2), 128 (1995). 